\title{
Modeling of Mixed Logical Dynamical (MLD) Model to Hybrid Three Tank System
}

\author{
Vinod Phani ${ }^{1}$, Cyril Joseph ${ }^{2}$, Satya Narayan Agarwal $^{3}$ \\ M.Tech, Electrical \& Electronics Engineering, Manipal University Jaipur, Jaipur ,India ${ }^{1}$ \\ Asst. Professor Department of Instrumentation \& Control Engineering, Manipal University, India ${ }^{2}$ \\ Asst. Professor, Electrical \& Electronics Engineering, Manipal University Jaipur, Jaipur ,India ${ }^{3}$
}

\begin{abstract}
In recent times the study of hybrid systems has caught the attention of many researchers. The motivation for the same is due to the fact that almost all the process systems in industries and laboratory exhibit hybrid dynamics. The term hybrid system is defined to describe the models, with both the continuous dynamics and discrete dynamics coexist and interact with each other. In this Project a three tank system is considered. The system can be modeled by using one of the hybrids modeling technique, mixed logical dynamical (MLD) model. A predictive control will also be designed for the developed model. The developed model will be verified and validated. MATLAB will be used as a tool to realize the model and the controller.
\end{abstract}

Keywords: Hybrid System, mixed logical dynamical, Three Tank System.

\section{INTRODUCTION}

Many practical systems consist of both continuous valued components and discrete valued components. The term hybrid system is used to describe models, where both continuous and discrete dynamics co-exist and interact. In this paper we proposed a method to model and control of systems interacting with the physical laws, logical rules, and operating constraints. Propositional logic is transformed into linear inequalities involving both integer variables and continuous variables. To control the integer variables and continuous variables we are adopting one technique called Mixed Logical Dynamical (MLD) systems in which it is described by linear dynamic equations subject to linear mixed-integer inequalities, i.e. inequalities involving both continuous and binary (or logical, or $0-1$ ) variables. These include physical/discrete states, continuous inputs and continuous/binary auxiliary variables.

In this paper, we proposed a MLD modeled to modeling and control of three-tank system with nonlinear system. The aim is to verify and define computational aspects of MLD method to deal with hybrid systems.

\section{Mixed logical dynamical system:}

Mixed Logic Dynamical (MLD) is one of the powerful tools in modeling of hybrid systems. Several aspects are dealing with MLD systems that can be formulated by using mixed integer optimization technique. In this modeling technique, each and every binary variable are assigned with some logic variable. If and only if the logic statement is true, then only the value of the binary variable one otherwise zero

$$
X_{j} \equiv \text { True } \leftrightarrow \delta_{j}=1
$$

Propositional logic of the system is transformed into equivalent linear

Inequalities. As shown below

$$
\mathrm{X}_{1} \vee \mathrm{X}_{2} \text { is equivalent to } \delta_{1}+\delta_{2} \geq 1
$$

And the statement is give as $\mathrm{X} \triangleq \mathrm{f}(\mathrm{n}) \leq 0$,
Where $f: R^{n} \rightarrow R$ is linear assume that $n \in X$ where $X$ is a given bounded set of values and defines as

$$
M \triangleq \max _{n \in X} f(n)
$$

$\mathrm{m} \triangleq$

$\min _{n \in X} f(n)$

The another basic principal of MLD modeling is the interaction between logical and dynamical variables it can be shown as follow

$$
[\mathrm{f}(\mathrm{n}) \leq 0] \leftrightarrow[\delta=1] \text { istrueiff }\left\{\begin{array}{c}
\mathrm{f}(\mathrm{n})=\mathrm{M}(1-\delta) \\
\mathrm{f}(\mathrm{n}) \geq \varepsilon+(\mathrm{m}-\varepsilon) \delta
\end{array}\right.
$$

2. Mixed logical dynamical system structure

The Modeling and controlling of systems can defined by physical laws, logical rules, and operating constraints called Mixed Logical Dynamical (MLD) systems. System model is defined by linear dynamic equations subject to linear inequalities involving both real and integer variables. Control action can be performed by using different schemes of optimal control (via dynamic programming) or predictive control. It can be implemented by Boolean algebra

The general form of an MLD system is given by the following expressions:

$$
\begin{gathered}
x(n+1)=A x(n)+B_{1} u(n)+B_{2} \delta(n)+B_{3} z(n) \\
y(n)=C x(n)+D_{1} u(n)+D_{2} \delta(n)+D_{3} z(n) \\
E_{2} \delta(n)+E_{3} z(n) \leq E_{1} u(n)+E_{4} x(n)+E_{5}
\end{gathered}
$$

Above equations describe about state update function, output function, and the set of inequalities in collects constraints on the system, as well as translations from logic proposition

$$
\begin{gathered}
\mathrm{X} \text { is the continuous and binary states } \\
x=\left[\begin{array}{c}
x_{n} \\
x_{l}
\end{array}\right], x_{n} \in R^{m}, x_{l} \in\{0,1\}^{n_{l}}
\end{gathered}
$$

$\mathrm{Y}$ is the continuous and binary states outputs

$$
y=\left[\begin{array}{l}
y_{n} \\
y_{l}
\end{array}\right], y_{n} \in R^{p_{c}}, y_{l} \in\{0,1\}^{p_{l}}
$$

$\delta_{l} \in\{0,1\}^{r_{l}}$ are the auxiliary binary variables $z \in R^{r}$ Are the auxiliary continuous variables 


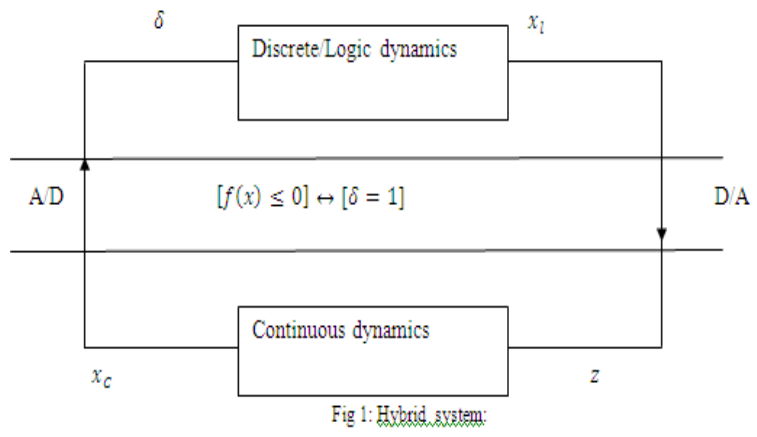

\section{HYBRID MODELING OF THREE TANK SYSTEM}

In the below system consists of three tanks with same cross-sectional area and that are supported with two independent identical pumps on Tanks' $q_{1}$ and $q_{2}$. The pumps provide the liquid flows from zero to maximum flow $q_{\max }$

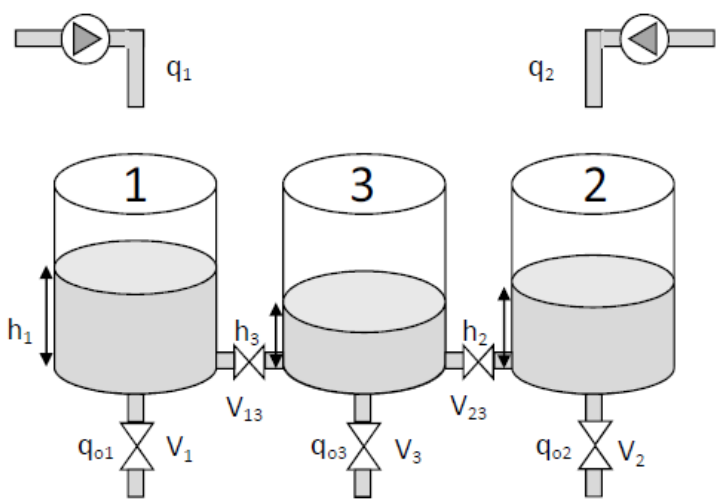

Each tank in the system is interconnected with each other by lower switching valves $, V_{13}, V_{23}$, and which are normally open and close conditions. The liquid levels in each tank are defined as $h_{1}, h_{2}$ and $h_{3}$ and each tanks can be measured with continuous valued level sensors.

In normal operation, the outflow of system is located at the middle tank; in other hand the valve $V_{3}$ is open. Other two valves $V_{1}$ and $V_{2}$ are close in normal condition are used to model failures in system

The differential equations for the Three Tank system can be written as follows

$$
\begin{gathered}
h_{1}=\frac{1}{A}\left(Q_{1}-Q_{13 v 13}-Q_{01}\right) \\
h_{2}=\frac{1}{A}\left(Q_{2}-Q_{32 v 32}-Q_{02}\right) \\
h_{3}=\frac{1}{A}\left(Q_{13 v 13}+Q_{23 v 23}-Q_{N 1}\right)
\end{gathered}
$$

$\mathrm{Q}$ is flow through valve and $\mathrm{A}$ is the cross sectional area of the each tank. Assuming that the flow through valve between tank 1 to tank3, and tank 2 to tank3 i.e. $V_{i 3}(\mathrm{i}=1,2)$ obeys Torricelli's law then the flow through $V_{i 3}$ is given as

$$
Q_{i 3 v i 3}=V_{i 3} a_{z} S_{i 3} \operatorname{sign}\left(h_{i}-h_{3}\right) \sqrt{\left|2 g\left(h_{i}-h_{3}\right)\right|}(\mathrm{I}=1,2)
$$

The flow through valves $V_{L 1}, V_{L 2}, Q_{N 3}$ is given as

$$
\begin{aligned}
Q_{L 1} & =V_{L 1} a_{z} S_{L 1} \sqrt{2 g h_{1}} \\
Q_{L 1} & =V_{L 2} a_{z} S_{L 2} \sqrt{2 g h_{2}} \\
Q_{N 3} & =V_{N 3} a_{z} S_{N 3} \sqrt{2 g h_{3}}
\end{aligned}
$$

$S_{L 1}, S_{L 2}, S_{N 3}$ Is the area of cross section of valves $V_{1}, V_{2}, V_{3}$. The outputs of the system is taken as the height of the liquid in each tank and denoted as follows

$$
\begin{aligned}
& y_{1}=h_{1} \\
& y_{2}=h_{2} \\
& y_{3}=h_{3}
\end{aligned}
$$

\section{CONCLUSION}

Hybrid systems provide a framework for describing processes system with continuous dynamics, discrete dynamics, and logic rules. There are several modeling methods are there to develop the hybrid systems, among them Mixed Logical Dynamical (MLD) systems is one of the method. Here we implemented Model Predictive Control (MPC) to control the hybrid systems. MPC is widely used in industry to solve the control problems with constraints.

\section{REFERENCES}

[1]. Y. Hammi, N. Zanzouri , M. Ksouri, "Modeling and Predictive Control of Hybrid System".

[2]. A. Bemporad and M. Morari. Control of system integrating logic, dynamics, and constraints

[3]. A. Bemporad, Hybrid Toolbox - User's Guide.

[4]. Bemporad. M, Borrelli. F and Morari. M, "Piecewise linear optimal controllers for hybrid systems,

[5]. Hammi. Y, Zanzouri. N and ksouri. M, Modeling and Predictive Control of Tow Tank System by MLD approach . The 4th International Conference on Circuits, Systems and Signals

[6]. Kvasnica. M, Grieder. P, Baotic. M and Morari. M Multi parametric toolbox

\section{HAND BOOK}

[1] H. Paul Williams, "Model Building in Mathematical Programming", A John Wiley \& Sons, Ltd., Publication, Fifth Edition. 